\title{
Der Wiener Börsenkrach und die Entstehung des modernen Aktienrechts
}

\section{Analysen zur Frage der Wahrnehmung von Nachhaltigkeitsdefiziten in den Wirtschaftskrisen der Moderne}

\author{
Sybille Kuhn
}

\section{Zusammenfassung}

Im Vorfeld der Ausarbeitung des allgemeinen deutschen Handelsgesetzbuchs 1861 setzt eine intensive Diskussion um das Aktienrecht ein. Da Aktiengesellschaften im Zentrum des Kollapses der Wiener Börse im Jahr 1873 stehen, kommt dieser Aktienrechtsdiskussion eine besondere Bedeutung zu, zumal anhand der zu erörternden Probleme wesentliche Aspekte aufscheinen, deren rechtliche Lösung eine Herausforderung für die Akteure darstellt. Darüber hinaus werden aber auch ethische Implikationen deutlich. Der Beitrag unternimmt den Versuch, die aktienrechtliche Diskussion aus der Perspektive der Akteure, hier im Besonderen der Handelskammern, zu skizzieren, um sie mit einer ethischen Grundlegung zu verknüpfen, wie sie aus den Quellen fassbar wird. Dieser Beitrag steht im größeren Zusammenhang eines Dissertationsprojekts, in dessen Rahmen am Beispiel der Weltwirtschaftskrisen von 1873, 1929-1933, der Asienkrise 1997/98 und der Immobilien- und Finanzkrise seit 2007 durch eine komparative Herangehensweise Wahrnehmungsmuster von Nachhaltigkeitsdefiziten in Wirtschafts- und Finanzkrisen der Moderne untersucht werden. Insbesondere wird der Frage nachgegangen, in welchen Bereichen die jeweiligen Akteure Defizite wahrnehmen, wobei als Akteure Wirtschaftsverbände im Zentrum der Untersuchung stehen.

\section{Fragestellung - Quellenlage - Gegenstand - Methode}

Die hier skizzierte Untersuchung, die im größeren Rahmen eines Dissertationsprojekts mit dem Titel Wahrnehmungsmuster von Nachhaltigkeitsdefiziten in Wirtschafts- und Finanzkrisen der Moderne steht, verfolgt am Beispiel der Welt- 
wirtschaftskrisen von 1873, 1929-1933, der Asienkrise 1997/98 und der Immobilienund Finanzkrise seit 2007 Diskurse zur Nachhaltigkeit, Werthaltigkeit und Ethik in Finanzsystemen aus der Perspektive der Verantwortungsträger der Wirtschaft, genauer: der Wirtschaftsverbände in Deutschland. Die zentrale Frage lautet: Werden auf der Basis der Analyse geeigneter Quellen unter Einbeziehung kommunikativer Strategien ethische Aspekte sichtbar und lassen diese sich als Diskurse fassen? Um sich dieser Perspektive so weit wie möglich anzunähern, dienen als Quellenbasis in erster Linie Dokumente der Verbände selbst: zum einen Verbandspublikationen, zum anderen aber auch Verlautbarungen der Verbände, wie sie in einschlägigen Tageszeitungen veröffentlicht wurden. Allerdings operieren diese Quellen auf der moralischen Ebene mit sehr weichen Begriffen wie, guten Sitten', ,Treu und Glauben' und ,Grundsätze eines ordentlichen Kaufmanns', mit ,Sittenwidrigkeit', ,Unwahrhaftigkeit', ,Unredlichkeit' und ,grober Verletzung des Gesetzes' - mit Begriffen also, die an Gewohnheit und Tradition anknüpfen und die zunächst an das moralische Gewissen der Akteure appellieren, dies jedoch in einem Bereich tun, der zugleich einer extremen Verrechtlichung unterliegt (Pfister, 2002: 51-61).

Die Quellen, die zur Auswertung vorliegen, stehen größtenteils in unmittelbarem zeitlichem Zusammenhang mit der jeweiligen Krise. Gelegentlich wird jedoch auf Quellenmaterial zurückgegriffen, das im Vorfeld der Krise zu verorten ist, denn vor allem für das 19. Jahrhundert gilt, dass ständig mit Krisen gerechnet wurde und daher eine Art Krisenbewusstsein vorausgesetzt werden kann und muss, das während der eigentlichen Krise den Horizont der Auseinandersetzung bildet.

Zudem gilt es bereits an dieser Stelle auf eine quellentechnische Schwierigkeit hinzuweisen, die aus der Einbeziehung des Wiener Börsenkrachs von 1873 und der sich daran anschließenden Gründerkrise in die Untersuchung resultiert: Die großen deutschen Wirtschaftsverbände gründen sich erst in der Folge dieser Krise (Ackermann, 2002: 134; Kiesewetter, 1989: 93-95).

Daher werden unterstützend Gutachten herangezogen, die von Handelskammern in Auftrag gegeben wurden. Da dies für diesen Zeitabschnitt gewissermaßen als Notmaßnahme vorgenommen werden muss, werden für die anderen Krisen, wo möglich, vergleichbare Gutachten flankierend konsultiert, um eine möglichst kohärente Betrachtungs- und Herangehensweise zu gewährleisten.

Die Zeit des Wiener Börsenkrachs fällt in eine wirtschaftspolitische Umbruchzeit, weil der Liberalismus zugunsten staatsinterventionistischer Eingriffe aufgegeben zu werden drohte. Diese antiliberale wirtschaftspolitische Tendenz stößt in den einzelnen Handelskammern auf unterschiedlichste Resonanz, was sich auch in den Stellungnahmen im Umfeld dieses Börsenzusammenbruchs widerspiegelt. Da als eine wesentliche Ursache für den Zusammenbruch die Liberalisierung des Aktiengesetzes angesehen wurde, widmet sich dieser Beitrag vorrangig diesem Aspekt, zumal 
die Diskussion um das Aktienrecht alle vier zu untersuchenden Wirtschaftskrisen gleichsam wie ein roter Faden durchzieht. Da jedoch eine Behandlung aller vier Krisen zu großen Raum einnehmen würde, liegt der Ausgangspunkt dieses Beitrags auf der Untersuchung des Wiener Börsenkrachs von 1873, zumal hier Aspekte aufscheinen, die bei allen weiter zu untersuchenden Krisen und insbesondere bei der Immobilien- und Finanzkrise seit 2007 erneut bedeutsam werden. Ziel des Beitrags ist mithin, Parallelen sowie signifikante Differenzen zwischen der ersten der hier zu betrachtenden Krise und den ihr im 20. Jahrhundert folgenden aufzuzeigen.

\section{$2 \quad$ Blicke auf den Wiener Börsenkrach (1873): Max Wirth und Adolf Soetbeer}

Die Nationalökonomen des 19. Jahrhunderts glaubten auf der Basis von Beobachtungen der wirtschaftlichen Entwicklung davon ausgehen zu können, dass es immer wieder aufgrund zyklischer Wellen zu Wirtschaftskrisen kommen werde. Wirtschaftskrisen waren demnach etwas, womit der in finanziellen Dingen bewanderte Fachmann rechnete, ja rechnen musste (Riesser, 1906; Riesser, 1971: 11f.). Zu den aufmerksamsten Beobachtern zählt der Nationalökonom und Journalist Max Wirth (1822-1900). Er war längere Zeit als Vorstand des volkswirtschaftlichen Kongresses und des Nationalvereins tätig und langjähriger Redakteur der Zeitschrift Arbeitgeber in Frankfurt am Main sowie in den Jahren von 1865 bis 1873 Direktor des Eidgenössischen Statistischen Büros in Bern. Seit 1874 war er neben seiner Tätigkeit als Redakteur der Neuen Freien Presse in Wien auch journalistisch für die Schlesische Presse tätig. Artikel von Wirth finden sich aber auch in Zeitschriften wie Die Grenzboten, die ein breiteres bildungsbürgerliches, vor allem an Literatur interessiertes Publikum ansprechen wollten. An dieser publizistischen Tätigkeit zeigt sich sein um Aufklärung bemühtes Anliegen, die Leser mit den wirtschaftspolitischen Zusammenhängen vertraut zu machen, das Bedürfnis, komplexe wirtschaftliche Zusammenhänge zu erklären und den Bereich der wirtschaftlichen Betätigung, vor allem den der Börse, einem größeren Publikum durchschaubarer werden zu lassen, um Ängste und Vorbehalte abzubauen und um eine kritische, sachliche Auseinandersetzung zu ermöglichen. Dieses bildungsbürgerliche Publikum galt es gerade in finanziellen Dingen aufzuklären, da es eine nicht unerhebliche Neigung zeigte, Barvermögen anzusparen und anzulegen, ohne jedoch fundierte Kenntnisse über die Zusammenhänge und damit über geeignete Anlageorte und -weisen zu besitzen (Riesser, 1971: 90ff.). 
Für das Fachpublikum verfasste Max Wirth einschlägige Standardwerke zu den wichtigsten wirtschaftlichen Problemen. Aus dieser Fülle seien neben seinem Handbuch des Bankwesens (1874) vor allem die Werke erwähnt, die im Umfeld des Wiener Börsenkrachs entstanden sind, so die Geschichte der Handelskrisen von 1858, die jedoch schon 1874 eine zweite erweiterte Auflage erfahren hat und in der Wirth die Erfahrungen mit dem Wiener Börsenkrach verarbeitet ${ }^{1}$; ferner Die Reform der Umlaufsmittel im deutschen Reiche. Ein Nachtrag zur Geschichte der Handelskrisen (1875), Österreichs Wiedergeburt aus den Nachwehen der Krisis (1876), Die Münzkrisis und die Notenbank-Reform im Deutschen Reiche (1874) sowie ein Referat Die österreichisch-ungarische Bankfrage (1875). Im Zentrum seiner Beobachtungen standen vor allem die sogenannten Umlaufsmittel, denn wenngleich sich Wirtschaftskrisen kaum völlig verhindern lassen, da sie zu schwer vorherzusehen sind, sah Wirth doch in der Steuerung der Umlaufsmittel ein probates Instrument, um so eine Krise abzumildern und im günstigsten Fall eine Panik zu verhindern. Diese Steuerung ist Aufgabe der Politik, insbesondere der Notenbank. Daher war Wirth, neben Adolf Soetbeer und Ludwig Bamberger, ein dezidierter Vorkämpfer für die Goldwährung und die Einrichtung der deutschen Reichsbank als alleiniger Notenbank.

Luzide zeichnet Wirth die Auswirkungen des Wiener Börsenkrachs nach, die er vor allem im Zusammenhang mit der Entwicklung der Umlaufsmittel vor dem Einsetzen des Zusammenbruchs der Wiener Börse sieht. So liegt seiner Ansicht nach eines der Grundübel und eine der Ursachen dieses Kollapses darin, dass die Preußische Bank nicht verhindert hatte, dass die Umlaufsmittel innerhalb kürzester Zeit, zwischen 1871 und 1873, allein in Deutschland durch die Prägung von Goldmünzen um 30 \% erhöht wurden, obwohl keinerlei wirtschaftliche Notwendigkeit dazu bestanden habe (Wirth, 1874b: 143). Die Folge dieser enormen Ausdehnung der Umlaufsmittel sei, dass das Gold nach Frankreich abfloss, um in der Diktion Wirths zu bleiben, was zur Folge gehabt habe, dass dort mit diesen Goldmünzen Rechnungen beglichen wurden und Waren in weit größerem Umfang als üblich aus Frankreich zu günstigen Konditionen nach Deutschland importiert wurden, der Handel demnach von dieser großen Ausdehnung der Umlaufsmittel profitierte, von den Konsequenzen im Devisenhandel ganz zu schweigen. ${ }^{2}$ Der Übergang zur Goldwährung in Deutschland hat Wirth zufolge die Krise deutlich beschleunigt,

1 In diesem Werk findet sich reichhaltiges statistisches Material, nicht nur zu sämtlichen Aktiengesellschaften, die an der Wiener Börse gehandelt wurden, sondern beispielsweise auch zu den Aktiengesellschaften des Deutschen Reichs.

2 Die Betrachtung dieser Frage wäre im hier verfolgten Zusammenhang durchaus lohnend, kann jedoch aus Platzgründen erst in einem Kapitel der Dissertation Berücksichtigung finden. 
ja verschärft, da die Doppelwährung nicht schnell genug beseitigt wurde (Wirth, 1874b: 143f.; Ziegler, 1996: 133f.).

Der langjährige Sekretär der Hamburger Commerzdeputation Adolf Soetbeer (1814-1892) war neben Ludwig Bamberger ,einer der Begründer der Goldwährung, ja vielleicht deren ältester praktischer Befürworter in Deutschland und bis ans Ende der 1880er Jahre ihr eifrigster Verfechter“, der jedoch früh die "Anzeichen einer Wendung der Entwicklung vor Augen zu sehen glaubte" (Halle, 1908: 405). ${ }^{3}$ Tatsächlich sollte Adolf Soetbeer mit seinem Brief vom 15. August 1891 an Ludwig Bamberger geradezu Max Wirth und dessen Überzeugung sekundieren, wie die Ausführungen zur Goldwährung unterstreichen:

Eine wesentliche Vermehrung des monetären Goldvorraths steht nicht zu erwarten [...], weil die Goldverwendung zu Schmucksachen (Uhren, Ketten, Ringen, Broschen usw.) und sonst zu industriellen Zwecken mit dem Anwachsen der Bevölkerung und des Wohlstandes in den meisten Culturländern stark zunimmt und die Goldproduction zu absorbiren die Tendenz hat. Allein es gewährt die Ausbildung und Ausdehnung des Bankwesens und der Papiercirculation andererseits einen entsprechenden Ersatz für den Bedarf der Culturländer an effectivem Gold auf Basis der Goldwährung - für gewöhnliche Zeiten. Für kurze Perioden allgemeinen Mißtrauens und außerordentlicher Erschütterungen des Credits und des Handels ist es m. E. nicht von entscheidender Bedeutung, ob die jährliche Goldgewinnung um einige Tausend Kilogramm abnimmt oder steigt, denn in solchen Ernstfällen wird Sistirung der Baarzahlungen, und zwar thunlichst gleich beim Beginn der Stockung, das alleinige Auskunftsmittel sein, wie dies z. B. 1870 in Frankreich mit Erfolg geschah. Abgesehen von solchen ganz ausnahmsweisen Eventualitäten, die ein Capitel für sich bilden, genügt m.E. der Goldbestand der großen Banken, um wegen der befürchteten Goldknappheit sich keinen Befürchtungen hinzugeben, da für absehbare Zeit die Vereinigten Staaten für ihren Geldumlauf, der riesig wächst, Silber in Aussicht nehmen. (Halle, 1908: 405).

Demzufolge gilt es nach Soetbeer, in einer Krise möglichst nicht in klingender Münze zu zahlen, vor allem nicht Gold auszumünzen, sondern Ersatz zu schaffen durch vermehrten Notenumlauf, Wechseldiscontierung und vergleichbare Maßnahmen.

Soetbeer, der zunächst als Latein- und Griechischlehrer an der Realschule in Hamburg unterrichtete, wurde von dem Hamburger Großkaufmann Vorwerck, der der Commerzdeputation 1841 als Präses vorstand, dazu animiert, statt sich nur mit

3 Da die Quellenlage zu Person und Rolle Adolf Soetbeers sehr dürftig ist und eine Monographie, die neuere Forschungsergebnisse berücksichtigt, ein Desideratum darstellt, kann der von Ernst von Halle verfasste Eintrag in der ADB im vorliegenden Kontext nach wie vor als grundlegend gelten. In neueren Publikationen findet Soetbeer allenfalls am Rande Erwähnung, obwohl er die wirtschaftlichen Zusammenhänge seiner Zeit überaus fundiert und kenntnisreich reflektiert und folglich für die Fragestellung dieser Arbeit von eminenter Bedeutung ist. 
der Römischen Geschichte zu beschäftigen, „sich lieber einmal mit einer für den Hamburger Handelsstand brennenden Frage" (Vorwerck o. J., zit. nach Halle, 1908: 400) zu befassen. Dabei ging es Vorwerck in erster Linie um die für den Handelsstand so lästige Einrichtung wie den Stader Elbzoll. Tatsächlich hatte sich der Altphilologe in der Folge mit seiner 1839 veröffentlichten Schrift Des Stader Elbzolls Ursprung, Fortgang und Bestand. Eine publicistische Darstellung „mit einem Schlage [...] als hervorragend befähigt zum Dienste für die Interessen ,Eines Ehrbaren Kaufmanns erwiesen" (Halle, 1908: 400). Da ihm die Kieler Universität daraufhin den Grad des „Doctor juris“ verlieh, stand einer Anstellung bei der Commerzdeputation nichts mehr im Weg, denn neben dem Kaufmann galt damals in Hamburg nur der Jurist „für öffentliche Leistungen als vollwerthig“(Halle, 1908: 400). Neben seiner amtlichen Tätigkeit verfasste er wirtschaftspolitische Publikationen, vor allem über den Hamburger Handel. Darin spiegelt sich auch das Vertrauen des Hamburger Handelsstandes, denn es war damals

noch nicht lange her, seit den Hamburger Zeitungen alle Veröffentlichungen über Angelegenheiten der Handlungen aufs strengste verboten waren, und gerade in der Commerzdeputation [hatten] maßgebende Elemente [...] in der Geheimhaltung aller commerziellen Dinge eine Hauptstütze des freistädtischen Geschäftsbetriebes zu sehen sich gewöhnt. (Halle, 1908: 400).

Daher waren, obwohl das Verbot nicht mehr galt, als Soetbeer mit seinen publizistischen Arbeiten begann, „doch im alten Hamburg Veröffentlichungen über Handelsangelegenheiten verpönt“ (Halle, 1908: 400f.). Soetbeers Integrität ist es zu verdanken, dass der Kaufmannsstand davon überzeugt werden konnte, „wie viel nützlicher und lehrreicher auf vielen Gebieten die Offenlegung der Dinge sei“ (Halle, 1908: 400f.), gerade in wirtschaftlichen Belangen. Da Soetbeer jedoch ein Aufstieg in der Commerzdeputation versagt blieb, war er bis 1872 deren Sekretär. Die Ursache dafür sieht Ernst von Halle (1908: 404) darin, dass wohl seine ablehnende Haltung „zur Frage der Erhaltung der Girobank und ihrer Währung" dazu beitrug, „daß er - weiterschauend als seine engeren Landsleute - bereits damals die Nothwendigkeit für die Hansestädte erkannte, ihre Stellung als wirthschaftlich völlig unabhängige Freihäfen aufzugeben. Er galt für einen Vertreter des ungeheuer unpopulären, ja für die öffentliche Stellung geradezu verhängnißvollen Standpunktes der Zollanschlußfreundschaft“. ${ }^{4}$

4 Aus diesem Grund nahm Soetbeer, „durch das erhebliche Vermögen seiner Frau finanziell unabhängig und wohlversorgt", in seinem 58. Lebensjahr eine Professur an der hamburgischen Landesuniversität Göttingen an (Halle, 1908: 404). 
Aufgrund seiner Erfahrung während und nach dem Wiener Börsenkrach blieb Soetbeer, der John Stuart Mill übersetzt und überhaupt erst im deutschen Sprachraum bekannt gemacht hatte, zeit seines Lebens ein Anhänger des Wirtschaftsliberalismus, auch wenn er sich der Probleme bewusst war, mit denen vor allem die Landwirtschaft, die für die Beibehaltung der Zölle eintrat, angesichts fallender Preise zu kämpfen hatte (Halle, 1908: 406). ${ }^{5}$ Zugleich brachte er den „arbeitenden Classen“ jedoch „stets einen Geist des patriarchalischen Wohlwollens" entgegen (Halle 1908: 406f.). Der Börsenkrach und dessen Folgen brachten Soetbeer zu der Überzeugung, dass die „politische Ökonomie“ von „natürlichen Gesetzen“ beherrscht werde, die der Wirtschaftsliberalismus am besten „zur Wirksamkeit bringen würde“ (Halle, 1908: 406f.). ${ }^{6}$

\section{$3 \quad$ Wahrnehmung und Struktur: die Diskussion um das Aktienrecht im Rahmen der Krise von 1873 und deren Folgen}

Mit der Gründerkrise 1873, die vor allem durch Spekulationen der Aktiengesellschaften hervorgerufen wird, entfaltet sich eine intensive Auseinandersetzung über die Rechtsform der Aktiengesellschaft, die zunächst mit dem Aktiengesetz des Jahres 1887 ihren Abschluss findet. Vor dem Hintergrund der zwölf Kammergutachten, die für den Wiener Börsenkrach und die Gründerkrise vorliegen, sollen hier exemplarisch zwei konträre Standpunkte skizziert werden: der der Handelskammer zu Hamburg und der der Handels- und Gewerbekammer Chemnitz (Hecht, 1874).

Die Handels- und Gewerbekammer Chemnitz plädiert dezidiert für eine „Einschränkung des Actien-Wesens und seiner sonstigen Privilegien" (Hecht, 1874: 172) und verlangt vom Gesetzgeber ein deutliches Eingreifen und die „Unterstellung der Aktiengesellschaften unter das deutsche Genossenschaftsrecht" (Hecht, 1874: 171). Demgegenüber betont die Handelskammer Hamburg, dass in Hamburg eine „in jeder Beziehung ungesunde Ausdehnung des Actienhandels [unbekannt]" sei, „weil bei uns durch die Gesetzgebung die Bevölkerung niemals an den Genuss scheinbarer und trügerischer gesetzlicher Garantien gewöhnt gewesen ist und,

5 An seinem Lebensabend konfronierte Soetbeer sich, da er „als Hypothekargläubiger einen großen Hof in der Nähe von Göttingen“ übernahm, ganz elementar mit den Herausforderungen der Landwirtschaft (Halle, 1908: 406).

6 Gestützt auf John Stuart Mill glaubte Soetbeer auch, „die Bestrebungen des Socialismus und Communismus erfolgreich bekämpfen“ zu können (Halle, 1908: 406f.). 
statt zum blinden Vertrauen auf schützende Gesetze, zum erprobten Unternehmen angeleitet worden ist“ (Hecht, 1874: 232). Deshalb gebe es auch „keinen Grund, sich irgendwie bei Revision des Actiengesetzes dem Mitgefühl mit geschädigten Actionären hinzugeben“; das Aktienwesen sei nur etwas für „Eingeweihte“, und die Revision des Aktiengesetzes nehme eine „durch und durch ungesunde Richtung“, wenn „angestrebt“ werde, „den kleinen Actionär gegen den grossen, das Publikum gegen die Banquiers, den Aussenstehenden gegen die Eingeweihten besser als bisher zu schützen“ (Hecht, 1874: 232). Das „innere Actien-Gesellschaftsrecht“ habe „eine strenge Durchführung des Grundsatzes, dass das Recht für die Wachsamen geschrieben wird, erfordet" (Hecht, 1874: 232). Die Unterweisung in wirtschaftlichen Dingen war für die Handelskammer Hamburg essentiell. So war es für sie selbstverständlich, dass, wer sich an solch einer Unternehmung wie einer Aktiengesellschaft beteiligen wollte, sich intensiv damit zu beschäftigen hatte; er hatte zu prüfen, wer die Gründer sind, ob sie vertrauenswürdig scheinen, über welche Vermögensverhältnisse sie verfügen, in was investiert werden soll etc. Die selbständige Prüfung sämtlicher Risikofaktoren lag demnach in der Pflicht des einzelnen; der Staat sollte nicht intervenieren. Ebenso lehnte die Handelskammer die Konzessionspflicht schon bei den Beratungen seit dem Jahr 1857, die zur Ausarbeitung des allgemeinen deutschen Handelsgesetzbuches (ADHGB) im Jahr 1861 führen sollten, entschieden ab, da sie darin die Gefahr sah, dass durch diese Pflicht der Bürger in einer Scheingewissheit gewiegt werde, die zu gründende Aktiengesellschaft sei absolut sicher und eine Aktie berge kein Risiko (Schubert, 1984). Zwar kämen Bankrott und Insolvenz immer vor, müssten jedoch auf den kleinen Kreis einer Handelselite beschränkt bleiben; daher müsse die Börse, zu der nicht jeder Zutritt habe, der ausschließliche Ort sein, an dem solche Geschäfte getätigt werden. Bezeichnenderweise war Hamburg viel weniger von der Gründerkrise betroffen als beispielsweise Berlin. Für die Hamburger Kaufleute war die Krise von 1857 eine Bewährungsprobe gewesen (Wirth, 1874a: 408-410). Die hier gesammelten Erfahrungen kamen ihnen bei der Gründerkrise zugute.

Nicht zuletzt im Umgang mit Wirtschaftskrisen unterscheiden sich demnach diese beiden Kammergutachten fundamental. Die Commerzdeputation in Hamburg ist dezidiert liberal: Derjenige, der in eine Aktiengesellschaft investiert, hat selbst das Risiko zu tragen, unter Umständen auch das des gesamten Verlusts seines investierten Kapitals, mehr jedoch nicht. Die Handels- und Gewerbekammer Chemnitz will hingegen die Aktiengesellschaften dem Genossenschaftsrecht unterwerfen, da sie vor allem in der ungenügenden Haftung ein ungeklärtes Problem sieht. Damit sind die beiden Pole der Auseinandersetzung innerhalb der zwölf Kammergutachten benannt, die übrigen Positionen liegen zwischen diesen Extremen. 
Dieser pointierte Vergleich der Gutachten macht deutlich, dass schon in dieser Zeit die Juristen zum einen Defizite in der mangelhaften Kontrolle durch den Aufsichtsrat sehen, da dieser eine Verschleierung der Bilanzen nicht wirksam unterbinden kann. Zum anderen liegen Defizite in der ungenügenden Haftung, vor allem aber auch in der schlechten Einzahlungsmoral der Gründer, durch die die Kapitalausstattung der Gründungen oft weit unter dem Nennwert der Aktien bleibt. Die Wahrnehmung dieser Defizite führt bei der zweiten Aktienrechtsnovelle von $1884 \mathrm{zu}$ Verschärfungen wie bestimmten Zahlungsverpflichtungen und Strafen für Bilanzverschleierungen. Schon allein die den Gründern auferlegten Zahlungsverpflichtungen sind bis zu einem gewissen Grade wirksam, da den neuen Bestimmungen Folge geleistet wird, und zeigen mithin, wie sensibel dieser Markt reagiert, zumal es gilt, verlorenes Vertrauen in die Anlageform der Aktiengesellschaft zurückzugewinnen (Münzel, 2006: 35 u. 55f.).

Die Diskussion über das Aktienrecht wird am Vorabend der Weltwirtschaftskrise erneut aufgegriffen, weil in Deutschland einschlägige Kartellgerichts- und Handelsgerichtsbeschlüsse die Tendenz zu Großunternehmen ebenso wie zu Zusammenschlüssen von Wirtschaftsunternehmen begünstigen. Denn durch die Neigung der deutschen Wirtschaft zur Bildung von „Interessengemeinschaften“, Kartellen und Zusammenschlüssen (Kipping, 2002: 281-283) ${ }^{7}$ - die Tendenz wird seit der Jahrhundertwende zunächst im Rückgriff auf die Erfahrungen mit der Kriegswirtschaft durch die Inflation noch verstärkt - entstehen durch Fusionen, die vor allem durch die Deutsche Bank begleitet werden (Gall et al., 1995: 233), insbesondere im Bereich der chemischen Industrie 1925 die I. G. Farben ${ }^{8}$ sowie im Bereich der Schwerindustrie 1926 die Vereinigten Stahlwerke (VSt) (Reckendrees, 2000: 149-249).

Doch auch auf dem Bankensektor selbst kam es in der Folge der Weltwirtschaftkrise zu aufsehenerregenden Zusammenschlüssen: 1929 fusionierte die Deutsche Bank mit der Disconto-Gesellschaft zur Deutschen Bank und Disconto-BankGesellschaft; 1931 wurde die DANAT-Bank nach ihrem Zusammenbruch am 17. Juni 1931 in die Dresdner Bank integriert. Neben der Commerzbank und der Berliner Handelsgesellschaft, die sich jedoch hauptsächlich in der Industriefinanzierung engagierte, gab es in Deutschland bis 1933 damit vier Großbanken (Gall et al., 1995: 258-308). Die neuen Konzernstrukturen in vielen Bereichen machen

7 Hierzu auch Frei et al., 2009: 61. Allgemein zu dieser Thematik Horn \& Kocka, 1979.

8 Liefmann (1930: 382) bezeichnet die 1925 gegründete Aktiengesellschaft der I. G. Farbenindustrie als "größten deutschen Trust“. Dazu auch Lindner (2005: 13-26), Stokes (2002: 221-258), ferner Plumpe (1990: 96-105). 
eine erneute Auseinandersetzung mit dem Aktienrecht erforderlich, um den neuen wirtschaftlichen Unternehmensformen in rechtlicher Hinsicht gewachsen zu sein.

An diesem Ringen um ein adäquates Aktienrecht, das auch intensiv in der Handelspresse begleitet und diskutiert wird, lässt sich zudem ablesen, in welchen Bereichen ein Defizit im Hinblick auf ein angemessenes, verantwortliches Handeln wahrgenommen wird. So „war es ein erklärtes Ziel der aktienrechtlichen Reformbemühungen der Weimarer Republik, die Rechte der Aktionäre zu stärken, um hierdurch das Vertrauen des Anlage suchenden Publikums in Aktien als Anlageform wieder herzustellen" (Mertens, 2007: 92). Mit dem Aktiengesetz von 1937 verschoben sich die Gewichte zugunsten einer Stärkung des Vorstands sowohl gegenüber dem Aufsichtsrat als auch gegenüber der Generalversammlung, die mit diesem Gesetz in ,Hauptversammlung' umbenannt wurde (Mertens, 2007: 92). Dennoch blieb das 1937 erlassene Aktiengesetz, obgleich vollständig reformiert, ein Provisorium, da es nicht in einer freien Wirtschaft und nicht im politischen Rahmen eines Rechtsstaats erlassen worden war (Mertens, 2007: 91-94). ${ }^{9}$

\section{$4 \quad$ Das Aktienrecht und die Grenzen der Politik}

Auf einen ersten Blick scheint die Gründerkrise noch ganz in ein nationales wirtschaftspolitisches Gefüge eingebunden zu sein. Aber auch hier, wie bei früheren Wirtschaftskrisen, beispielsweise 1857, als die amerikanische Wirtschaftskrise die Hamburger Börse tiefgreifend erschütterte (Ahrens, 1978), zeigt sich durch die in Österreich von den französischen Kontributionszahlungen an das Deutsche Reich ausgelösten hochgespannten Erwartungen, dass die österreichische Wirtschaft hoffte, deutsche Anleger würden ihr Geld in Österreich investieren. Aufgrund dieser Erwartung wurde die Gründung von Aktiengesellschaften beflügelt (Neuwirth, 1874: 3; Soetbeer, 1874: 41)..$^{10}$

Auf wirtschaftspolitischer Ebene war von Bedeutung, dass die für Aktiengesellschaften und für Kommanditgesellschaften auf Aktien seit 1861 bestehende Konzessionspflicht am 11. Juni 1870 mit dem Inkrafttreten der ersten Aktienrechtsnovelle aufgehoben wurde. Damit war auch die staatliche Aufsicht abgeschafft, an deren Stelle

9 Zu diesem Komplex auch Münzel (2006: 44 u. 139-147) sowie Bayer \& Engelke (2007).

10 Der Nationalökonom Neuwirth, 1873 von der Brünner Handelskammer in das österreichische Abgeordnetenhaus entsandt, spricht angesichts der fünf Milliarden Goldfrancs Kriegsentschädigung von „Zündstoff“ (Neuwirth, 1874: 3). Hierzu auch Ferguson (2002: 267). 
privatrechtliche Normativbedingungen entstanden: Jede Aktiengesellschaft musste nunmehr neben einem Vorstand und der Generalversammlung einen Aufsichtsrat haben und in das Handelsregister eingetragen werden (Mertens, 2007: 91). Dieser Aufsichtsrat vertrat jedoch eher die Funktion eines Verwaltungsrats - das Problem der Kontrolle war damit noch nicht befriedigend gelöst, weil deren Notwendigkeit und die gerade darin liegende eigentliche Bedeutung des Aufsichtsrats noch nicht im Blickfeld lagen. $\mathrm{Zu}$ ergänzen ist jedoch, dass es sehr wohl einer Konzession durch eine staatliche Behörde bedurfte, sobald eine Aktiengesellschaft gegründet werden sollte, deren Unternehmung von gesamtgesellschaftlicher Relevanz war, beispielsweise große Infrastrukturvorhaben, zu denen in erster Linie Eisenbahngesellschaften und der Kanalbau zählten. In der tatsächlichen Umsetzung entsprach die Erteilung der Konzession allerdings oftmals lediglich einer Formsache und führte daher eher zur Entlastung der staatlichen Behörden (Lieder, 2007: 323f.: 327; Schubert \& Hommelhoff, 1985). Daher trugen selbst Konzessionierungsverfahren kaum dazu bei, den Anlegern zu garantieren, dass die bei ihrer Gründung mit einer Konzession ausgestatteten Aktiengesellschaften kein Risiko bargen. De facto waren diese Gründungen oft äußerst gewagt und wiesen durchaus eine risikoreiche Kapitalbeschaffungskonstruktion auf. ${ }^{11}$ Als Nebeneffekt ergab sich zudem seit Bestehen der Konzessionspflicht, dass die Aktiengesellschaften selbst mit Konzessionen handelten (Kiesewetter, 1989: 82). Deshalb war die Kritik der Hamburger Commerzdeputation an diesem Verfahren durchaus berechtigt, weil aufgrund einer Informationsasymmetrie zu wenige sich tatsächlich informieren konnten und daher zu große Risiken eingingen, deren Folgen so nicht abzuschätzen waren (Lieder, 2007: 325).

Gemeinhin wird in der Liberalisierung des Aktienrechts eine der Ursachen für die Gründerkrise gesehen. Demgegenüber ist jedoch zu betonen, dass die damaligen Wirtschaftsvertreter, etwa die Hamburger Kaufmannschaft, gerade in dem trügerischen Schein der vermeintlichen staatlichen Kontrolle, sei es durch die Konzessionierung oder durch die Normativregelung, ein großes Risiko sehen. Es besteht vor allem darin, dass sich die Anleger auf diese Regelungen blind verlassen, ohne die finanzielle Basis der Gründung einer eingehenden Prüfung zu unterziehen. Andere Wirtschaftsvertreter wie die Chemnitzer Handels- und Gewerbekammer sehen in der Institution der Aktiengesellschaft per se eine nicht zu kontrollierende Rechtsform; sie plädieren für die Unterbindung dieser Gesellschaftsform, obgleich sie im Grunde unerlässlich für die Kapitalbeschaffung großer Unternehmungen ist (Lieder, 2007: 324).

11 Als berühmtester Fall gilt derjenige des ,Eisenbahnkönigs` Strousberg (Kiesewetter, 1989: 80-82). 
Mit dem Entstehen von Großunternehmen schon um die Jahrhundertwende, vor allem aber nach dem Ersten Weltkrieg, wird eine Aktienrechtsreform immer dringender, da die Konstruktion der nun entstehenden Riesenunternehmen Vorbild sind die Trusts in den USA - nicht mehr mit dem Aktiengesetz des Jahres 1884 vereinbar zu sein scheint. So wird, wie in Abschnitt III gezeigt, in der Weimarer Republik die Diskussion zum Aktienrecht erneut aufgegriffen, die ihren Niederschlag nicht nur in den stenographischen Aufzeichnungen der von der Reichsregierung eingesetzten Kommission zur Reform des Aktienrechts findet, sondern auch in Publikationen der Verbände, die geradezu in die Offensive gehen, sowie in detailreichen, ebenfalls von den Verbänden beeinflussten Artikeln in den Handelsteilen einschlägiger Tageszeitungen. ${ }^{12}$

Der Deutsche Juristentag, aber auch die Handelskammern erarbeiten Vorschläge zur als überfällig wahrgenommenen Reform des Aktienrechts. Denn die deutsche Privatwirtschaft hatte Gesellschaftsformen entwickelt, die im Falle einer Wirtschaftskrise die Situation eher zu verschärfen als zu erleichtern drohten. Die Verantwortlichen jedoch nehmen gerade diese Gesellschaftsformen offenbar nicht als problematisch wahr, sondern sehen beispielsweise in den Kartellen eine Garantie für eine dem Markt angemessene Preisentwicklung. Die Aktie selbst erfährt ebenfalls eine Entwicklung dahingehend, dass sich ihr eine Sonderform hinzugesellt, die sogenannte Mehrstimmenaktie. Mit dieser Sonderform hat der Vorstand in den Gesellschaften ein viel größeres Gewicht und kann so das Abstimmungsverhältnis in der Generalversammlung entscheidend beeinflussen. Das Instrument der Mehrstimmenaktie ist durch das Aktiengesetz von 1884, das diese Aktienform noch gar nicht kennt, nicht gedeckt. Sie wird erst um die Wende zum 20. Jahrhundert erfunden und soll nun durch eine Aktienrechtsreform in den 1930er Jahren legalisiert werden (Mertens, 2007: 94f.).

Das angestrebte Ideal der Aktienrechtsreform der Weimarer Zeit hat zum Ziel, für nahezu alle denkbaren Fälle ein Gesetz zu erlassen. Als Kehrseite dieser umfassenden Aktienrechtsreform samt der Einführung der Wirtschaftsprüfung entstand jedoch die Gefahr, dass die Akteure - Gründer, Vorstand, Aufsichtsrat respektive Verwaltungsrat, Aktionäre wie Anleger ohne Stimmrecht - gerade durch die weitreichende Verrechtlichung aus ihrer Verantwortung entlassen würden. Der Aufsichtsrat beispielsweise glaubte, die zuvor ihm selbst obliegende Verantwortung nun an den Wirtschaftsprüfer delegieren zu können. Tatsächlich aber hatte und

12 Erwähnt seien etwa die Handelsteile der Kölnischen Zeitung, der Frankfurter Zeitung, der Vossischen Zeitung, der Berliner Börsen-Zeitung und des Berliner Börsen-Couriers, aber auch die der Deutschen Allgemeinen Zeitung, das „Hausorgan der Ruhrindustrie“ (Frei, Ahrens, Osterloh \& Schanetzky, 2009: 110). 
hat bis heute diese Instanz ausschließlich unterstützende und beratende Funktion, keine Entscheidungsbefugnis und somit auch keine Verantwortung für getroffene Entscheidungen. ${ }^{13}$

Zugleich sollte mit dem neuen Berufsstand des Wirtschaftsprüfers eine Instanz geschaffen werden, die dem Kontrollorgan, etwa dem Aufsichtsrat, zur Seite gestellt werden könnte, um die Glaubwürdigkeit der Bilanzen zu garantieren, denn die Intransparenz der publizierten Daten galt damals als ein großes Problem (Jenkis, 1989: 16f.). ${ }^{14} \mathrm{Da}$ die Bilanzen in Deutschland als Betriebsgeheimnis definiert wurden, ${ }^{15}$ waren sensible Daten allenfalls eingeweihten Bankspezialisten zugänglich, weshalb Max M. Warburg, Mitglied der Handelskammer Hamburg und von 1924 bis 1933 Mitglied des Generalrats der Reichsbank, als einer der ersten die Notwendigkeit erkannte, die Institution der Wirtschaftsprüfung in der deutschen Wirtschaft zu etablieren (Kleßmann 1999: 62; Rosenbaum \& Sherman, 1978: 156f.). Während der Weimarer Republik war dies zunächst eine vertrauensbildende Maßnahme, um amerikanischen Investoren entgegenzukommen. ${ }^{16}$ Interessanterweise findet die Etablierung der Wirtschaftsprüfer als Folge der Weltwirtschaftskrise ihrerseits eine Parallele bei der Entwicklung der Corporate Compliance. Das Compliance Management System erfuhr durch den Sarbanes-Oxley Act (SOA) von 2002, der nach den großen Finanzskandalen von Enron und Worldcom erlassen wurde, einen neuen Impuls und stellt vor allem deutsche Unternehmen, deren Aktien an der New Yorker Börse gehandelt werden, vor große Herausforderungen. Der SOA ist auch wirksam für amerikanische Tochtergesellschaften, die beispielsweise in Deutschland ihren Sitz haben. Da das deutsche Recht, im Unterschied etwa zu dem der USA, kein Unternehmensstrafrecht kennt, bestimmt hier der SOA eine Art ethischer Richtschnur des finanzpolitischen Handelns, insbesondere für Großunternehmen (Klöckner, 2009).

13 Hierzu grundlegend Jenkis (1989).

14 Der Berufsstand der Wirtschaftsprüfer wurde 1931 geschaffen, um spektakuläre Unternehmenszusammenbrüche, wie sie sich in den späten 1920er Jahren ereignet hatten, „für alle Zeiten auszuschließen" (Jenkis, 1989: 17). Mit seiner Rede von diesen Zusammenbrüchen spielt Jenkis auf den Streichholzmonopolisten Ivar Kreuger an, auf die Brüder Georg Carl, Friedrich und Heinrich Lahusen, Inhaber der Norddeutschen Wollkämmerei und Kammgarnspinnerei AG mit Sitz in Bremen (Nordwolle), sowie auf den Unternehmer Ludwig Katzenellenbogen und dessen Berliner Brauerei Schultheiss-Patzenhofer.

15 In den USA beispielsweise war dies nicht der Fall, und entsprechend wurde dort die in Deutschland übliche Praxis auch scharf kritisiert (Liefmann, 1930: 402).

16 De facto brachten die amerikanischen Investoren oftmals ihre eigenen Wirtschaftsprüfer mit, um die Bilanzen einzusehen (Ritschl, 2002: 11). 


\section{$5 \quad$ Konsequenzen}

Bei der Untersuchung der möglichen Auslöser und Folgen des Wiener Börsenkrachs und der Gründerkrise wurde auf eine Konsequenz schon in zeitgenössischen Quellen, vor allem österreichischer Provenienz, hingewiesen: Es wurde als Defizit wahrgenommen, dass jedes Geldinstitut alle Bankgeschäfte betreiben konnte, unabhängig von seiner finanziellen Ausstattung, so dass viele Anleger einen Verlust ihres Vermögens erlitten und von weiteren Anlagen Abstand nahmen. Dieses Defizit stellt nach Joseph Neuwirth die Ursache für den Geldentzug dar, der aus diesem Misstrauen gegenüber dem Finanzsektor resultierte und der für die Realwirtschaft zunehmend eine Schwierigkeit darstellte, weil kaum noch Aktien gekauft wurden (Dirninger, 2000: 31, 34ff.; Neuwirth, 1874: 5). Zudem hatte dieses Defizit in Österreich zur Folge, dass verstärkt in sichere Anlagen investiert und die Entwicklung von Sparkassen, mithin eine Stärkung der Kommunen, vorangetrieben wurde. Sparkassen galten als sicheres Bankinstitut, da sie nicht in unsolide, „schwindelhafte Gründungen“ (Feldkircher Zeitung, 1874) investiert und sich von der Börse ferngehalten hatten (Dirninger, 2000: 30; Feldkircher Zeitung, 1874).

Demgegenüber wurden in den USA andere Konsequenzen aus der Weltwirtschaftskrise gezogen. Unter F. D. Roosevelt wurde 1933 für den Bankensektor mit dem Inkrafttreten des Glass-Steagall Act die Trennung von Geschäfts- und Investitionsbanken eingeführt. Allerdings wurde diese Trennung in den 1990er Jahren zunehmend aufgeweicht und unter Clinton 1999 endgültig aufgehoben. Ökonomen, unter anderen auch Robert Shiller (2012), sehen darin eine der Ursachen für die Krise von 2007 (Nikolov, 2000: 139).

Eine weitere Konsequenz aus der Weltwirtschaftskrise ist die neu geregelte Frage der Haftung. Bis 1970 schrieb die New Yorker Börse ihren Mitgliedern vor, in der Unternehmensform der Personengesellschaft verfasst zu sein, also einer unbeschränkten Haftung zu unterliegen, so dass, wenn eine Gesellschaft verklagt wird, jeder einzelne Gesellschafter persönlich haftbar ist und folglich bei einem Konkurs auch alles verlieren kann (Shiller, 2012: 240). Hier scheint die schon von der Chemnitzer Handels- und Gewerbekammer im 19. Jahrhundert formulierte Forderung verwirklicht worden zu sein, dass Aktiengesellschaften sich dem Gesellschaftsrecht der Genossenschaften zu unterwerfen haben und bei den Gründern einer Aktiengesellschaft eine strenge Haftungsregel anzuwenden ist, die über das investierte Kapital hinausgeht. Mitte der 1980er Jahre wurde dieser Weg verlassen.

Dies stellte die Wirtschaft wieder vor die Situation, mit der sie schon seit dem Inkrafttreten des ADHGB 1861 gerungen hatte. Allerdings schien im Unterschied zum 19. Jahrhundert das Bewusstsein für die Risiken deutlich weniger ausgeprägt 
zu sein. Denn bereits in der Kommission, die mit der Ausarbeitung der ADHGB und speziell des Aktiengesetzes betraut gewesen war, war

darauf hingewiesen [worden], dass ein wirtschaftlicher Fehlschlag eines Unternehmens nicht immer auf mangelhafter Gründung oder Missmanagement beruht, sondern nicht selten ,große Vermögensverluste [...] durch Ereignisse und Konjunkturen entstehen, deren Eintritt und Folgen sich bei aller Erfahrung und Umsicht nicht im Voraus erkennen lassen`. (Hadding \& Kießling, 2003: 186f.).

Zumindest in diesen Fällen gehe der in den Beratungen geäußerte Einwand ins Leere,

die Aktionäre könnten Haftungsrisiken durch Kontrolle der Geschäftsführung oder Beschränkung der Vertretungsmacht des Vorstands auf Geschäfte, die aus dem Unternehmensvermögen beglichen werden können, begrenzen. Demgegenüber trete der durchaus notwendige Schutz des Publikums in den Hintergrund. [...]. Letztlich sei eine Aktiengesellschaft mit unbeschränkter Gesellschafterhaftung wertlos, da begüterte Aktionäre Anteile an unvermögende Dritte weiterveräußern und so den Gläubigern die angestrebte Haftungsgrundlage rückwirkend wieder entziehen könnten. (Hadding \& Kießling, 2003: 186f.).

Für die Kommission war demnach selbst eine unbeschränkte Gesellschafterhaftung keine Gewähr dafür, dass die Aktionäre bei einem Konkurs die Möglichkeit besäßen, den Gesellschaftern gegenüber Forderungen geltend zu machen. Da diese ihre Anteile schon zuvor veräußern könnten, sei die notwendige Sicherung des Geschäftsverkehrs nur durch andere Maßnahmen, als bisher im Gesetz vorgesehen, zu erreichen. Dies ist die Geburtsstunde des „Grundsatzes der realen Kapitalaufbringung und Kapitalerhaltung im Recht der Kapitalgesellschaften“ (Hadding \& Kießling, 2003: 186f.). ${ }^{17}$

\section{$6 \quad$ Fazit und Ausblick: Wirtschaftskrisen zwischen Liberalismus und Neoliberalismus}

Im Bewusstsein des hohen Risikos, das es im Wirtschaftsleben einzugehen gilt, waren die - meist noch ganz dem Liberalismus verhafteten - Akteure am Ausgang des 19. Jahrhunderts bemüht, sich jeglicher staatlichen Eingriffe zu erwehren. Der Liberalismus rechnet mit der vollen Verantwortlichkeit des wirtschaftlich Han-

17 Der hier angeschnittenen Frage von Investition und Kapitalerhaltung wird sich ein eigenes Kapitel der geplanten Dissertation widmen. 
delnden: Er muss die Konsequenzen seines wirtschaftlichen Agierens selbst tragen und kann diese Verantwortung nicht auf Dritte übertragen. Neuwirth (1874: 5f.) stellt fest, dass schon im Vorfeld des Wiener Börsenkrachs, im Jahr 1872, sich das „capitalbesitzende, solide Anlagen suchende Publicum“ längst zurückgezogen hatte und „der hohle Bau noch eine gute Weile hielt“, da die „Überspeculation durch die unerfahrene, vom leichten Gewinne jederzeit magnetisch angezogene Masse der Bevölkerung und ein bis dahin ungekannter, waghalsiger Creditmissbrauch" das Ganze noch einige Zeit zusammenhielt.

Wenngleich die Weltwirtschaftskrise 1929 in Deutschland die Wirtschaftsverbände vor besondere, neue Herausforderungen stellte, sei doch hier in aller Kürze angemerkt, dass diese Verbände sich als sehr anpassungsfähig zeigten und sich rasch auf die politischen Gegebenheiten einstellen konnten, auch darauf bedacht, die Eingriffe des Staates in wirtschaftliche Belange auf ein Minimum zu reduzieren.

Wie gefährlich demgegenüber die enge Bindung von Staat und Wirtschaft werden konnte, zeigt paradigmatisch die Gelsenberg-Affäre am Ende der Weimarer Republik als eine der Folgen der Weltwirtschaftskrise in Deutschland. Durch die Übernahme des Aktienpakets des Industriellen Friedrich Flick gelangte der Staat zu einer „Mehrheitsbeteiligung am größten deutschen Montankonzern“, was „die komplizierte Machtbalance zwischen der Schwerindustrie und dem verarbeitenden Gewerbe aus dem Gleichgewicht brachte" (Frei et al., 2009: 113). ${ }^{18}$ Daher war die Empörung über dieses Geschäft nicht nur bei den Montanindustriellen besonders groß (Bähr, 2010; Frei et al., 2009: 112), sondern auch bei den Verbänden, was erklärt, warum Gustav Krupp von Bohlen und Halbach, der „den zweitgrößten Montankonzern nach dem Stahlverein, aus dem nun unvermittelt das größte Privatunternehmen seiner Branche geworden war“, vertrat, „in seiner Funktion als Präsident des Reichsverbands der Deutschen Industrie Flick zur Rede stellen wollte“ (Frei et al., 2009: 113). Albert Vögler, der Direktor des Stahlvereins (Vst), sah sich als Vertreter Flicks schweren Vorwürfen innerhalb der Ruhrlade, des einflussreichsten informellen Zirkels der Wirtschaftselite der Weimarer Republik, ${ }^{19}$ ausgesetzt, da Flick, statt sich aus der Verantwortung zu stehlen und dem Staat seine Anteile anzubieten, zunächst innerhalb der Wirtschaft nach einer Lösung für sein

18 Hierzu auch Gall, 1995: 309.

19 Bei der Sitzung der Ruhrlade am 26. Juni 1932, bei der über den Verkauf des Gelsenberg-Aktienpakets, das aus einem Volumen von 100 Millionen Mark bestand, diskutiert wurde, waren neben Gustav Krupp von Bohlen und Halbach Peter Klöckner, Paul Reusch, Generaldirektor der Gutehoffnungshütte (Haniel), Fritz Springorum, Generaldirektor der Hoesch AG, und Albert Vögler (Vereinigte Stahlwerke (Vst), in den Quellen oft als Stahlverein bezeichnet) anwesend (Frei, 2009: 113). 
Finanzierungsproblem hätte suchen müssen, so dass die Trennung von Staat und Wirtschaft gewährleistet gewesen wäre (Frei et al., 2009: 113, 98-117).

Ein nur sehr summarischer Blick auf die beiden letzten großen wirtschaftlichen Erschütterungen, die Immobilien- und Finanzkrise seit 2007 sowie die gelegentlich als deren Vorbote bezeichnete Asienkrise 1997/98 ${ }^{20}$, zeigt, dass sie in die Zeit fallen, die maßgeblich geprägt ist durch das Signum des Neoliberalismus. Dieser ist geleitet von der Annahme, dass sich die Akteure rational verhalten und dass der Markt eine Ordnungsfunktion übernimmt, die alles in einem ausbalancierten Verhältnis hält. Der Staat habe sich aus diesem System herauszuhalten, da die Märkte vollkommen seien. ${ }^{21}$ Wie wenig jedoch gerade dieses System auf den Staat verzichten kann, zeigte spätestens und exemplarisch der Zusammenbruch von Lehman Brothers im Jahr 2008, der nicht zuletzt auch als Folge dessen gesehen werden kann, dass der Staat zunehmend jenen Ordnungsrahmen aufgelöst hat, den er nach der Weltwirtschaftskrise in den USA geschaffen hatte. ${ }^{22}$

Das Sich-Herauslösen der Banken aus dem Dienst für die Wirtschaft, genauer, ihre zunehmende Distanz zur Realwirtschaft und ihr gleichzeitiges Eintreten in die Finanzwirtschaft, eröffnet den Banken einen immensen Spielraum, den zu beherrschen jedoch zunehmend schwieriger wird. Die beiden jüngsten Kollapse, die Asienkrise 1997/98 und die Immobilien- und Finanzkrise seit 2007, zeigen jenem System eine Grenze, wenngleich diese noch verschiebbar zu sein scheint. Jedoch deuten sie schon an, dass ein globales neoliberales Wirtschaftssystem sich kaum mehr mit nationalen Ordnungsrahmen fassen lässt, dass es vielmehr nach einem supranationalen Regelsystem verlangt. Dieses muss sich allerdings einer verfassungsmäßigen rechtsstaatlichen Legitimation stellen und letztlich unterwerfen.

$20 \mathrm{Zu}$ dieser Diskussion Stiglitz (2007) und Jomo (2008).

21 Hierzu vor allem Friedman (2010); die amerikanische Originalausgabe dieses Werks erschien bereits 1962 unter dem Titel Capitalism and Freedom. Herber \& Engel (1991: 70f.) führen die Börsen als ein Beispiel für vollkommene Märkte an, während etwa Akerlof \& Shiller (2009: 18ff.) diesem theoretischen Modell gegenüber eine große Skepsis zum Ausdruck bringen.

22 Die Bundesrepublik beschritt nach 1945 einen etwas anderen Weg. Hier fiel die Entscheidung zugunsten des Ordo-Liberalismus, der maßgeblich dazu beitrug, dass die Banken noch nicht zu Investmentbanken wurden, sondern sich in den Dienst der Wirtschaft zu stellen hatten. 


\section{Literatur}

Ackermann, V. (2002). Ein Zentrum der Eliten-Kommunikation - Industrie-Club e. V. Düsseldorf. In: J.-O. Hesse, C. Kleinschmidt \& K. Lauschke (Hrsg.), Kulturalismus, Neue Institutionenökonomik oder Theorievielfalt. Eine Zwischenbilanz der Unternehmensgeschichte (pp. 133-149). Essen: Klartext.

Ackermann, V. (2006). Treffpunkt der Eliten. Die Geschichte des Industrie-Clubs Düsseldorf. Düsseldorf: Droste.

Ahrens, G. (1978). Die Überwindung der hamburgischen Wirtschaftskrise von 1857 im Spannungsfeld von Privatinitiative und Staatsintervention. Zeitschrift des Vereins für Hamburgische Geschichte, 64: 1-29.

Akerlof, G. \& Shiller, R. J. (2009). Animal Spirits. Wie Wirtschaft wirklich funktioniert. Frankfurt a. M.: Campus.

Bähr, J. (2010). Paul Reusch und Friedrich Flick. Zum persönlichen Faktor im unternehmerischen Handeln. In: H. Berghoff, J. Kocka \& D. Ziegler (Hrsg.), Wirtschaft im Zeitalter der Extreme. Beiträge zur Unternehmensgeschichte Österreichs und Deutschlands. Im Gedenken an Gerald D. Feldman (pp. 275-297). München: Beck.

Bähr, J., Drecoll, A. \& Gotto, B. (2008). Der Flick-Konzern im Dritten Reich. Hrsg. v. Institut für Zeitgeschichte München-Berlin i.A. der Stiftung Preußischer Kulturbesitz. München: Oldenbourg.

Bayer, W. \& Engelke, S. (2007). Die Revision des Aktienrechts durch das Aktiengesetz von 1937. In: W. Bayer \& M. Habersack (Hrsg.), Aktienrecht im Wandel. Bd. 1: Entwicklung des Aktienrechts (pp. 619-669). Tübingen: Mohr Siebeck.

Dirninger, C. (2000). Sparkassen und Staatsintervention. In: R. Tilly (Hrsg.), Bankenkrise in Mitteleuropa im 19. und 20. Jahrhundert (pp. 13-48). Stuttgart: Steiner.

Feldkircher Zeitung (1874). Ausgabe vom 11. Februar.

Ferguson, N. (2002). Die Geschichte der Rothschilds. Propheten des Geldes (2. Aufl.). Stuttgart: DTV.

Frei, N., Ahrens, R., Osterloh, J. \& Schanetzky, T. (2009). Flick. Der Konzern, die Familie, die Macht. München: Blessing.

Friedman, M. (2010). Kapitalismus und Freiheit (7. Aufl.). München: Piper (1. Aufl. 1962).

Gall, L., Feldman, G. D., James, H., Holtfrerich, C.-L. \& Büschgen, H. E. (1995). Die Deutsche Bank 1870-1995. München: Beck.

Hadding, W. \& Kießling, E. (2003). Anfänge des deutschen Aktienrechts: Das Preußische Aktiengesetz vom 9. November 1853. In: J. Eckert (Hrsg.), Der praktische Nutzen der Rechtsgeschichte. Hans Hattenhauer zum 8. September 2001 (pp. 159-190). Heidelberg: Müller.

Halle, E. v. (1908). Soetbeer, Adolf Georg. In: Historische Commission bei der königl. Akademie der Wissenschaften (Hrsg.), Allgemeine Deutsche Biographie (ADB), Bd. 54 (pp. 399-408). Leipzig: Duncker \& Humblot.

Hecht, F. (1874). Credit-Institute auf Actien und auf Gegenseitigkeit. Bd. 1: Das Börsenund Actienwesen der Gegenwart und die Reform des Actien-Gesellschafts-Rechts. Mannheim: Schneider.

Herber, H. \& Engel, B. (1991). Volkswirtschaftslehre für Bankkaufleute (5. Aufl.). Wiesbaden: Gabler. 
Horn, N. \& Kocka, J. (Hrsg.) (1979). Recht und Entwicklung der Großunternehmer im 19. und frühen 20. Jahrhundert. Wirtschafts-, sozial- und rechtshistorische Untersuchungen zur Industrialisierung in Deutschland, Frankreich, England und den USA. Law and the Formation of the Big Enterprises in the 19th and Early 20th Centuries. Studies in the History of Industrialization in Germany, France, Great Britain and the United States. Göttingen: Vandenhoeck \& Ruprecht.

Jenkis, H. (1989). Die Wirtschaftsprüfer im Konflikt zwischen Prüfung und Beratung. Ursprung, Entwicklung, Probleme. Berlin: Duncker \& Humblot.

Jomo, K. S. (2008). Lektionen aus der Ostasienkrise. In: K. Küblböck \& C. Staritz (Hrsg.), Asienkrise: Lektionen gelernt? Finanzmärkte und Entwicklung (pp. 127-142). Hamburg: VSA-Verlag.

Kiesewetter, H. (1989). Industrielle Revolution in Deutschland 1815-1914. Frankfurt a. M.: Suhrkamp.

Kipping, M. (2002). Aspekte der Beziehungen zwischen Staat und Unternehmen. Lektionen für die Bedeutung des internationalen Vergleichs in der Unternehmensgeschichte. In: J.-O. Hesse, C. Kleinschmidt \& K. Lauschke (Hrsg.), Kulturalismus, Neue Institutionenökonomik oder Theorievielfalt. Eine Zwischenbilanz der Unternehmensgeschichte (pp. 275-288). Essen: Klartext.

Kleßmann, E. (1999). M. M. Warburg \& Co. Die Geschichte eines Bankhauses. Hamburg: Dölling und Galitz.

Klöckner, S. (2009). Informationspflichten und Haftung der Organmitglieder börsennotierter Aktiengesellschaften. Ein Vergleich Deutschland/USA. Baden-Baden: Nomos.

Lieder, J. (2007). Die 1. Aktienrechtsnovelle vom 11. Juni 1870. In: W. Bayer \& M. Habersack (Hrsg.), Aktienrecht im Wandel. Bd. 1: Entwicklung des Aktienrechts (pp. 318-387). Tübingen: Mohr Siebeck.

Liefmann, R. (1930). Die Unternehmungen und ihre Zusammenschlüsse. Bd. II: Kartelle, Konzerne und Trusts. Stuttgart: Moritz.

Lindner, H. (2005). Hoechst. Ein I.G.-Farben-Werk im Dritten Reich (2. Aufl.). München: Beck.

Mertens, B. (2007). Das Aktiengesetz von 1937 - unpolitischer Schlussstein oder ideologischer Neuanfang? Zeitschrift für neuere Rechtsgeschichte, 29: 88-117.

Münzel, M. (2006). Die jüdischen Mitglieder der deutschen Wirtschaftselite 1927-1955. Paderborn: Schöningh.

Neuwirth, J. (1874). Bank und Valuta in Oesterreich-Ungarn 1862-1873. Bd. 2: Die Speculationskrisis von 1873. Leipzig: Duncker \& Humblot.

Nikolov: R. (2000). Die Rolle der Banken im Finanzsystem. Eine komparative Analyse der Bankensysteme in Deutschland und den USA. Marburg: Tectum.

Pfister, U. (2002). Unternehmerverhalten im 19. Jahrhundert jenseits der Bürgerlichkeit. In: J.-O. Hesse, C. Kleinschmidt \& K. Lauschke (Hrsg.), Kulturalismus, Neue Institutionenökonomik oder Theorievielfalt. Eine Zwischenbilanz der Unternehmensgeschichte (pp. 51-61). Essen: Klartext.

Plumpe, W. (1990). Die IG-Farbenindustrie-AG. Wirtschaft, Technik und Politik 1904-1945. Berlin: Duncker \& Humblot.

Reckendrees, A. (2000). Das „Stahltrust“-Projekt. Die Gründung der Vereinigten Stahlwerke A.G. und ihre Unternehmensentwicklung 1926-1933. München: Beck.

Riesser, J. (1906). Zur Entwicklungsgeschichte der deutschen Großbanken mit besonderer Rücksicht auf die Konzentrationsbestrebungen (2. Aufl.). Jena: Fischer. 
Riesser, J. (1971). Die deutschen Großbanken und ihre Konzentration im Zusammenhang mit der Entwicklung der Gesamtwirtschaft in Deutschland (4. Aufl.). Glashütten i.T.: Auvermann (1. Aufl. 1912).

Ritschl, A. (2002). Deutschlands Krise und Konjunktur 1924-1934. Binnenkonjunktur, Auslandsverschuldung und Reparationsproblem zwischen Dawes-Plan und Transfersperre. Berlin: Akad.-Verlag.

Rosenbaum, E. \& Sherman, A. J. (1978). Das Bankhaus M. M. Warburg \& Co. 1798-1938 (2. Aufl.). Hamburg: Christians.

Schubert, W. (Hrsg.) (1984). Protokolle der Commission zur Berathung eines allgemeinen deutschen Handelsgesetz-Buches. Bd. 1. Frankfurt a. M.: Keip.

Schubert, W. \& Hommelhoff, P. (1985). Hundert Jahre modernes Aktienrecht. Eine Sammlung von Texten und Quellen zur Aktienrechtsreform 1884 mit zwei Einführungen. Berlin: de Gruyter.

Shiller, R. J. (2012). Märkte für Menschen. So schaffen wir ein besseres Finanzsystem. Frankfurt a. M.: Campus.

Soetbeer, A. (1874). Die fünf Milliarden. Betrachtungen über die Folgen der großen Kriegsentschädigung für die Wirthschaftsverhältnisse Frankreichs und Deutschlands. Berlin: Lüderitz.

Stiglitz, J. E. (2007). Die Asienkrise zehn Jahre später. https://www.project-syndicate.org/ commentary/the-asian-crisis-ten-years-after/german. Zugegriffen: 09.04.2015.

Stokes, R. G. (2002). Von der I.G. Farbenindustrie AG bis zur Neugründung der BASF (1925-1952). In: W. Abelshauser (Hrsg.), Die BASF. Eine Unternehmensgeschichte (pp. 221-358). München: Beck.

Wirth, M. (1874a). Geschichte der Handelskrisen (2. Aufl.). Frankfurt a.M.: Sauerländer (1. Aufl. 1858).

Wirth, M. (1874b). Die Goldausfuhr und die Münzreform. Die Grenzboten, 4: 140-147.

Ziegler, D. (1996). Eisenbahnen und Staat im Zeitalter der Industrialisierung. Die Eisenbahnpolitik der deutschen Staaten im Vergleich. Stuttgart: Steiner.

Open Access Dieses Kapitel wird unter der Creative Commons Namensnennung - Nicht kommerziell 2.5 International Lizenz (http://creativecommons.org/licenses/by-nc/2.5/deed. de) veröffentlicht, welche für nicht kommerzielle Zwecke die Nutzung, Verbreitung und Wiedergabe in jeglichem Medium und Format erlaubt, sofern Sie den/die ursprünglichen Autor(en), den Titel des Werks und die Quelle ordnungsgemäß nennen, einen Link zur Creative Commons Lizenz beifügen und im Falle einer Abwandlung durch einen entsprechenden Hinweis deutlich erkennbar machen, dass Änderungen vorgenommen wurden.

Die in diesem Kapitel enthaltenen Bilder und sonstiges Drittmaterial unterliegen ebenfalls der genannten Creative Commons Lizenz, sofern sich aus der Abbildungslegende nichts anderes ergibt. Sofern das betreffende Material nicht unter der genannten Creative Commons Lizenz steht und die betreffende Handlung nicht nach gesetzlichen Vorschriften erlaubt ist, ist auch für die oben aufgeführten nicht-kommerziellen Weiterverwendungen des Materials die Einwilligung des jeweiligen Rechteinhabers einzuholen. 